\title{
Historia de los comienzos de la laparoscopia en Colombia
}

\author{
Jorge E. Medina Murillo*
}

En los albores del siglo XXI, la endoscopia se considera un auxiliar indispensable en la mayoría de las especialidades quirúrgicas, debido a que cada día se busca utilizar procedimientos menos invasivos, ambulatorios en lo posible y que ofrezcan una rápida recuperación a los pacientes. Hoy se cuenta con instrumental sofisticado y con profesionales idóneos del equipo de salud para la realización de estos procedimientos. No era lo mismo en Colombia en los comienzos de la década de los setenta, cuando en muy pocos centros hospitalarios se realizaba endoscopia ginecológica. Por amable solicitud que me hizo el Dr. Gilberto Martínez, voy a tratar de revivir algunos recuerdos sobre los comienzos de la laparoscopia en Colombia.

Para hablar de laparoscopia vale la pena hacer una breve reseña sobre su desarrollo en el mundo y algunos hechos importantes que precedieron en Colombia su empleo.

Fue Ott en Petrogrado en 1901 fue el primero en hacer una inspección óptica de la cavidad abdominal. Lo hizo con la paciente en Trendelemburg, a través de colpotomía y ayudado con separadores vaginales, utilizó un espejo de cabeza con luz incandescente. Kelling en 1902 publicó un artículo titulado "Uber Oessophagoskopie, Gastroskopie und Kolioskopie". Utilizando un perro anestesiado, pasó un trocar por la pared abdominal e introdujo un cistoscopio de Nitze, diseñado desde 1878.

Más tarde en 1910, Jacobeus fue el primero en la técnica de "Laparoscopia" en seres humanos y el procedimiento sólo lo utilizaba en pacientes con ascitis. Ruddock publicó su primer trabajo en 1934, llamando el procedimiento "peritoneoscopia", y usando el cistoscopio de McCarthy con algunas modificaciones (1-2).

Debo mencionar aquí, que utilizando el equipo descrito por Ruddock de iluminación distal, en 1951, los doctores Arecio Peñaloza en Bogotá, Alberto Jamis en Barranquilla y Tomás Quevedo en Medellín hicieron las primeras laparoscopias para observar la cavidad abdominal superior, principalmente la superficie hepática (3-4).

En los Estados unidos, Decker en 1944 introdujo la culdoscopia, visualizando los órganos pélvicos por vía vaginal a través del fondo de saco de Douglas con la paciente en posición genupectoral. Ellos encontraron la

\footnotetext{
** Miembro Fundador de la Sociedad Colombiana de Médicos Endoscopistas. Miembro de la Asociación Americana de Ginecólogos Laparoscopistas.
}

peritoneoscopia incómoda para visualizar los órganos pélvicos porque se les interponían las asas intestinales, mientras que utilizando la posición genupectoral en pacientes seleccionados, obtenían con la punción del fondo de saco posterior un neumoperitoneo espontáneo que permitía buena visualización de los órganos pélvicos. En 1946 Palmer, en Francia pregoniza la laparoscopia, llamándola celioscopia ginecológica, enfatizando en la utilidad de colocar una cánula intrauterina para la mejor manipulación de los órganos pélvicos. Palmer estaba familiarizado con la culdoscopia, pero él encontró mayores ventajas en la vía abdominal que en la vaginal (2). Clyman en 1963 describió el culdospocipo operatorio que permitía hacer a través de una colpotomía posterior, con la ayuda visual del culdoscopio, pequeñas intervenciones, como cauterización de implantes de endometriosis, liberación de adherencias, aspiración de quistes de ovario y cirugía de trompas como esterilización tubárica (5). El estuvo como profesor invitado en el Congreso Colombiano de Obstetricia y Ginecología celebrado en Bucaramanga en 1971.

La laparoscopia fue promovida por ginecólogos europeos en las décadas de los cincuentas y los sesenta. Tuvo poca aceptación en los Estados Unidos porque allá preferían la culdoscopia, pero en 1968 aparecen publicaciones de Fear, Cohem, Siegler y Bereny sobre las ventajas de la laparoscopia comparada con la culdoscopia (2). Para la década de los" setenta se inieia una amplia difusión del procedimiento. El grupo de John Hopkins desarrolla un programa internacional para preparar médicos en actividades de planificación familiar, incluyendo técnicas de esterilización.

En 1972 el Dr. Jordan Philips fundó la Asociación Americana de Ginecólogos Laparoscopistas, entidad que cada día tiene más miembros. Hoy es considerada como la rectora en la endoscopia pélvica a nivel mundial. Cuenta en la actualidad con más de 5.000 miembros (1).

Ya en Colombia, desde la década de 1950 el Dr. Rodulfo Camero utilizaba el culdoscopio de Decker con fines diagnósticos en el estudio de problemas de esterilidad. El Dr. Fernando del Corral comenzó a emplear el culdoscopio de Clyman en 1964, (6-7) y los Drs. Acosta Bendek en Barranquilla (8) y Medina en Bogotá (9) han reportado sus experiencias principalmente para el estudio del factor tuboperitoneal en infertilidad.

La AVS Asociación para Esterilización Voluntaria realizó una importante reunión internacional en Ginebra entre el 26 de febrero y el 1o. de marzo de 1973. A esta 
reunión asistieron invitados por la AID distinguidos especialistas de varias ciudades del país. El Dr. Fernando Tamayo, Presidente de Profamilia y posteriormente de la IPPFF ocupaba un lugar destacado entre los directivos de dicha reunión.

Como resultado de las experiencias presentados por delegados de diferentes paises, los participantes colombianos regresaron decididos a estimular la práctica de estos procedimientos y vencer las resistencias, principalmente por parte de la Iglesia Católica.

En agosto 3 de 1973 la Sociedad Colombiana de Obstetricia y Ginecología realizó un Simposio sobre Esterilización Femenina Voluntaria en la ciudad de Bogotá, donde se trataron temas novedosos para la época como aspectos socio demográficos, indicaciones médicas, aspectos psicológicos, técnicas, aspectos legales y aspectos éticos. Participaron como conferencistas los Drs. Guillermo López Escobar, Fernando Sánchez Torres, Humberto Rosselli, Ricardo Rueda, Antonio José Cancino, Jaime Bernal Cuéllar y el padre Alfonso Llano y actuó como coọrdinador Jorge Medina (10).

En Colombia, los progresos en el estudio de la pareja estéril y la necesidad de tomar conciencia de buscar respuestas al impacto de la explosión demográfica, creó en la década de los sesenta un ambiente propicio para el desarrollo de la endoscopia pélvica.

\section{Laparoscopia en Colombia}

Como ya se dijo al hablar de los antecedentes en 1951 el Dr. Arecio Peñaloza en Bogotá, el Dr. Tomás Quevedo en Medellín y el Dr. Alberto Jamis en Barranquilla, comienzan a utilizar la peritoneoscopia de los americanos o laparoscopia de los europeos con un equipo de Ruddock de iluminación distal (3-4).

Sin embargo sólo hasta que los ginecólogos la empezaron a utilizar se generalizó su empleo en la práctica médica de finales del siglo.

En noviembre de 1972 el Dr. Gonzalo Echeverry director de Profamilia, al aceptar el curso ofrecido en la Universidad de John Hopkins, envió a el Dr. Miguel Pulido a este curso que duró un mes, fue eminentemente teórico, pero en marzo de 1973 vino a Colombia el Dr. Cliford Wheeles, director del programa internacional de planificación familiar de la Universidad de John Hopkins y dictó un curso práctico de una semana en el Hospital Infantil Lorencita Villegas de Santos, asistieron los Drs. Pulido y Jorge Michelsen como dicentes, y algunos otros médicos de Bogotá como observadores. La técnica preconizada por ellos utilizaba una sola punción y anestesia local convirtiendo el procedimiento en ambulatorio.

El Programa de esterilización laparoscópica lo inició Profamilia con el Dr. Miguel Pulido en mayo de 1973. Así lo relata el Dr. Gonzalo Echeverry: "Una vez terminada la capacitación del Dr. Pulido, Profamilia quiso establecer el programa de esterilización laparoscópica en un hospital de Bogotá, con el fin de tener el medio quirúrgico que garantizara al máximo la seguridad del procedimiento.

La timidez de algunos directores de hospitales y el riesgo de posibles conflictos con la Iglesia Católica hicieron abortar el proyecto. Profamilia decidió entonces asumir toda la responsabilidad y establecer el programa de laparoscopia en la propia clínica piloto, instalando un quirófano equipado para operar con todas las garantías, hasta el punto de que en una visita de seguimiento que hicieron los médicos de John Hopkins, se manifestaron sorprendidos al comprobar que el quirófano del Centro Piloto presentaba más facilidades de trabajo que el suyo en Baltimore" (11). Con este programa Colombia fue el primer país de Sur América en dar al servicio, no sólo la laparoscopia como método de esterilización femenina, sino el primero en establecer una clínica de esterilización Voluntaria tanto femenina como masculina.

En el Centro Piloto de Profamilia las laparoscopias fueron realizadas durante los primeros seis meses por el Dr. Pulido, luego él enseña al Dr. José M. Briceño y en 1974 inician el entrenamiento formal de médicos: los primeros capacitados fueron profesionales de Profamilia, entre ellos los doctores Aníbal Castañeda de Medellín, Saulo Muñoz de Cali, Fabio Durán de Bucaramanga, Alfredo Peña Olgiastri de Barranquilla, Abel Villegas de Pereira, y algunos otros (12). Después continuaron entrenando médicos del Ministerio de Salud y profesionales de otros países Latinoamericanos. El doctor Pulido publicó sus primeras experiencias en la Revista Colombiana de Obstetricia y Ginecología (13-14).

A finales de 1973 el mismo grupo de John Hopkins dictó un curso en el Instituto Materno Infantil, para capacitar médicos del Instituto y del Hospital San Rafael de Girardot con el fin de extender los programas a diferentes regiones. Los Drs. Guillermo López Escobar, Eduardo Cáceres Alvarez y Luis Armando Muñoz publican en 1974 su experiencia con las primeras 534 laparoscopias realizadas en este programa, también en forma ambulatoria y con anestesia local (15). Estos seminarios de adiestramiento y el trabajo de Laparoscopia fueron financiados por la Fundación Pathfinder de Boston.

El 4 de julio de 1974 el Dr. Aníbal Castañeda realiza la primera esterilización laparoscópica en Medellín (17) y en ese mismo mes el Dr. Fernando Del Corral compra el primer Laparoscopio para utilizarlo en Cali y trae a los Dis. L. Wharton y J. Jordan para desarrollar curso de adiestramiento para los colegas caleños (16). En septiembre de 1975 organiza otro curso con el auspicio del ISS; vinieron como profesores invitados de Estados Unidos los Drs. Cliffors R. Wheeles y R.R. Raechele y algunos docentes y médicos de diferentes ciudades de Colombia. Asistieron más de treinta especialistas de diferentes ciudades aprovechando esta oportunidad para fundar la Sociedad Colombiana de Médicos Endoscopistas. El Dr. Fernando del Corral fue elegido como su primer presidente. En Manizales inician el programa la laparoscopia en 1976 (18).

Entre las personas que han contribuido a la utilización masiva en programas de esterilización no sólo en Colombia sino en otros paises es necesario mencionar a el Dr. Gabriel Manzi, quien en 1981 empezó a realizar las laparoscopias introduciendo el trocar sin previo pneumoperitoneo. En diciembre de 1992 publicaron la experiencia con esta técnica, la cual está basada en la tracción de la pared abdominal con pinza de campo, 
incisión de piel 2-3 cms por encima del ombligo y paso del trocar sin previo neumoperitoneo. Durante este período habían realizado 136.627 esterilizaciones tubáricas con muy reducido número de complicaciones, tres lesiones vasculares, una de aorta, otra de vena cava y otra del mesosalpinx, que pudieron ser reparadas durante el mismo acto quirúrgico y además se presentaron cuatro lesiones del intestino delgado, y en una de ellas la paciente falleció semanas más tarde en sepsis (19).

El empleo de la laparoscopia a nivel de práctica privada también se fue desarrollando en forma paralela. El Dr. Otto Uscher siendo docente en el Hospital San Ignacio fue invitado a fines de 1973 a visitar algunos centros médicos de Estados Unidos estando allí decidió comprar un laparoscopio junto con los doctores Alberto Angulo y Elkin Lucena. Después de un año de llevar su laparoscopio de hospital en hospital, deciden en 1975 instalar un centro de laparoscopia ambulatoria en una casa adaptada para ese fin. Practicaban además de laparoscopias estudios de esterilidad, y vincularon como andrólogo al Dr. Sergio Bonilla. Durante este tiempo realizaron más de mil laparoscopias, sin ninguna mortalidad y con mínima morbilidad, relata el Dr. Uscher (20). Después el grupo se disuelve y el Dr. Lucena funda en 1977 el Centro Colombiano de Fertilidad y Esterilidad CECOLFES, donde en 1985 fue fecundado con éxito el primer bebé probeta de latinoamérica.

En 1973 el Dr. Arturo Aparicio Laserna, cuando estaba haciendo su residencia en el Hospital San José, asistió al curso del Instituto Materno Infantil, demostró entusiasmo por la laparoscopia, participó en la mayoría de las que realizaban en el hospital San José. Así adquirió destreza para ejecutar cirugía a través del laparoscopio, y mientras la mayoría de los ginecólogos utilizaban la técnica para fines diagnósticos, y principalmente para realizar esterilizaciones tubáricas, él liberaba adherencias, aspiraba quistes de ovario o fulguraba focos de endometriosis. En 1982 abrió su propio centro de laparoscopia, importó un equipo Storz con las más modernas especificaciones para la época, y así logró hacer laparoscopia operatoria con la tecnología más avanzada de ese tiempo (21). Aquí vale la pena mencionar la técnica que él desarrolló para fulguración ovárica para inducir ovulación, la cual publicó en 1982, (22) dos años antes de que apareciera en enero de 1984, el artículo del Dr. Halvard Gjönnaess en Fertility and Sterility (23) sobre su experiencia en 62 mujeres con síndrome de ovario poliquístico tratadas por electro cauterio a través del laparoscopio, el autor de ese artículo encontró que después de tres meses la ovulación reapareció en el $92 \%$ de las pacientes, hubo ciclos regulares en el $86 \%$, los embarazos ocurrieron en 24 de 35 pacientes que deseaban tener un hijo. Aparicio trató 15 pacientes, de ellas 14 ovularon durante los primeros 7 meses. Obtuvo seis embarazos, 5 a término y un aborto. Actualmente este método es una alternativa en el manejo de anovulación por síndrome de ovarios poliquísticos.

En la década de los ochenta el entusiasmo por laparoscopia fue notorio, su empleo se extendió a los principales centros universitarios, incluyéndose como parte formal del entrenamiento durante la residencia. Se empezó a observar también aumento de instituciones privadas dedicadas al estudio y tratamiento de la infertilidad.

En 1986 vinieron como profesores invitados a Cali a dictar un curso de aplicación del LASER en ginecología incluyendo técnicas endoscópicas con video laparoscopia los Drs. Carl Herbert y Wayne Maxon de la Universidad de Vanderbilt en Tennessee y el Dr. Adien Alvarez de la Universidad de Antioquia. Estas innovaciones en la práctica de la laparoscopia, sin lugar a duda, dieron un gran impulso a la laparoscopia operatoria en el país.

En el XX Congreso Colombiano de Obstetricia y Ginecología realizado en Medellín en marzo de 1996 en el correlato presentado por los Drs. Alfredo Gómez, Jaime Machicado y Guido Parra, (24) informaron sobre 91 centros repartidos por todo el país en donde se realizan laparoscopias, en muchos de ellos ya se encuentra completamente actualizado el sistema endoscópico con diversos tipos de energía, incluyendo el empleo del LASER y se observa una tendencia al aumento hacia el empleo de Video-Laparoscopia.

Al escribir la historia de la laparoscopia en Colombia quise únicamente presentar un resumen sobre hechos, que a mi juicio vale la pena recordar sobre sus comienzos, sin entrar en detalles sobre las dos últimas cuando ya se convirtió en un procedimiento usual en la práctica ginecológica.

La tendencia de la práctica quirúrgica en todas las especialidades es realizar procedimientos lo menos invasivo posible, buscando menor traumatismo para los pacientes y más rápida recuperación. Así pues, el continuo desarrollo de la endoscopia ginecológica nos ofrece un espacio importante en el ejercicio de la especialidad para el siglo XXI.

\section{BIBLIOGRAFIA}

1. Chatman DL., Cohen MR. "History of Endoscopy" manual of Endoscopy, The American Association of Gynecologic Laparoscopists, 1990; 1-10.

2. Cohen Melvin R. "Laparoscopy, culdoscopy and Gynecography" Major problems in Obstet. and Gynecol. 1970; 1: 12.

3. Peñaloza A. "Historia de la Medicina. Servicio de Gastroenterológía y endoscopia digestiva del hospital San José de Bogotá". Rev. Col. Gastroent. 1992; 7(4): 227-232.

4. Peñaloza A. "Endoscopia digestiva en Colombia. Pasado, presente y futuro" Rev. Col. Gastroent. 1997; 12(2): 67-72.
5. Clyman MJ. "A new panculdoscope-diagnostic, photographic, and operative aspects". Obstet. Gynecol. 1963; 21: 343.

6. Del Corral F., Artuza y Guzmán CA. "La culdoscopia como método auxiliar de diagnóstico en ginecología. Rev. Col. de Obst. y Ginec. 1964; 15: 429.

7. Del Corral F. "Ligadura y sección tubárica por culdoscopia” Rev. Col. de Obst. y Ginec. 1973; 24(5): 329-332.

8. Acosta Bendek E. "La culdoscopia en la práctica ginecológica". Rev. Col. de Obst. y Gineco. 1975; 26(4): 231-236.

9. Medina J. Culdoscopia, Análisis de 52 casos. Tribuna Médica No. 533 A3, 1972. 
10. Simposio sobre esterilización Femenina Voluntaria. Revista Col. de Obst. y Gineco. 1974; 25(3)

11. Echeverry G. "Contra viento y marea". 25 años de planificación Familiar en Colombia. A.C.P.E. Primera edición, septiembre 1991, pág. 156.

12. Pulido M. Com., personal.

13. Pulido M., Briceño JM. "Esterilización femenina voluntaria por el método de laparoscopia" Rev. Col. Obst. y Ginec. 1974; 25(5): 329-334.

14. Pulido M. "Esterilización femenina voluntaria por el método de laparoscopia 1.000 casos" Rev. Col. de Obst. y Ginec. 1975; 26(4): 225-230.

15. López Escobar G., Cáceres E., Muñoz LA. "Esterilización femenina ambulatoria por laparoscopia" Revista Col. de Obst. y Gineco. 1974; 25(3): 211-220.

16. Del Corral F. Comunicación personal.

17. Ríos B. Comunicación personal.
18. Lenis Nicholls N., Cifuentes JH., Calle M., Esparza C. "Laparoscopia diagnóstica en Ginecología” Rev. Col. Obst. y Ginec. 1982; 33(5) 289-296.

19. Bioló RG., Manzi GB. "Safe Laparoscopic Surgery: Tubal ligation Without Prior Pneumoperitoneum". Surgical Laparoscopy \& Endoscopy 1995; 4(5): 1-6.

20. Uscher O. Comunicación personal.

21. Aparicio A. Comunicación personal.

22. Aparicio Laserna A., García Pacheco R. "Fulguración ovárica ¿Inductor de la Ovulación? Ensayo preliminar”. Revista Colombiana de esterilidad Año II 1982; 1(4-5).

23. Gjönnaess H. "Polycistic ovarian syndrome treated by ovarian electrocautery through the laparoscope". Fertility and Sterility 1984 41(1): 20-25.

24. Gómez A., Machicado J. y Parra G. "Endometriosis, laparoscopia, histeroscopia". Correlato XX Congreso Nacional de Obstetricia y Ginecología, Medellín, marzo de 1996. 